rates were $74 \%, 18 \%$, and $6 \%$, respectively. Oral corticosteroids were used in $56 \%$ of PBO and $55 \%$ of BARI pts at baseline; pts continued use throughout the studies. The differences in clinical efficacy between BARI $4 \mathrm{mg}$ and PBO at 12 weeks was similar regardless of the number or type of csDMARDs concomitantly used (Table) or the concomitant use of corticosteroids (data not shown). The rates of serious adverse events and discontinuation due to adverse events were comparable regardless of the number or type of csDMARDs used (Table) or corticosteroid use.

ACR20/50/70 $=20 \%, 50 \%$, and $70 \%$ improvement in American College of Rheumatology criteria; csDMARDs=conventional synthetic disease-modifying antirheumatic drugs; DAS28-ESR=Disease Activity Score 28-erythrocyte sedimentation rate; $\mathrm{MTX}=$ methotrexate; SDAI=Simple Disease Activity Index.

Conclusions: BARI has demonstrated clinical safety and efficacy in a wide range of pts, regardless of the number of concomitant csDMARDs or concomitant use of corticosteroids.

References:

[1] Taylor PC et al. Arthritis Rheumatol 2015;67(S10):3927-3928.

[2] Dougados M et al. Ann Rheum Dis 2017;76:88-95.

Disclosure of Interest: A. Kavanaugh Consultant for: Eli Lilly and Company, C. Helt Employee of: Eli Lilly and Company, D. Muram Employee of: Eli Lilly and Company, J. Alam Employee of: Eli Lilly and Company, V. Arora Employee of: Eli Lilly and Company, A. L. Pinto Correia Employee of: Eli Lilly and Company, I. de la Torre Employee of: Eli Lilly and Company, R. van Vollenhoven Grant/research support from: Abbvie, Amgen, BMS, GSK, Pfizer, Roche, UCB, Consultant for: Abbvie, Biotest, BMS, Celgene, Crescendo, GSK, Janssen, Eli Lilly and Company, Merck, Novartis, Pfizer, Roche, UCB, Vertex

DOI: 10.1136/annrheumdis-2017-eular.1342

\section{THU0079 EVALUATION OF SYNOVITIS IN THE FOOT AND THE ANKLE NOT INCLUDED IN THE 28-JOINT DISEASE ACTIVITY SCORE USING ULTRASONOGRAPHY AND HISTOLOGICAL FINDINGS IN THE PATIENTS WITH RHEUMATOID ARTHRITIS}

\section{A. Abe, H. Ishikawa. Rheumatology, Niigata Rheumatic Center, Shibata, Japan}

Background: In the treatment of rheumatoid arthritis (RA), the early diagnosis and early medical treatment via tight control have become increasingly important with the advent of biological therapy. Ultrasonography (US) for the affected joints enables the evaluation of synovial hypertrophy, effusion and bone erosion in real time. US is a reliable method that can detect more erosive sites than radiography. US is now utilized widely and is a reliable tool of rheumatologists for diagnosing RA and evaluating the disease activity.

Objectives: This study was conducted to clarify the relationship between the systemic disease activity, local disease activity using US and a synovial histopathological evaluation.

Methods: Between March 2011 and September 2015, 668 joints were surgically treated on the whole, and synovial biopsies were performed at the time of surgery. Among them, a total of 118 toes and 21 ankles, which are not included in the 28-joint disease activity score (DAS28), were investigated. Just before surgery, the US probe was placed on the dorsal aspect of the ankle and/or the toes to evaluate the activity of local synovitis. The maximum grade of power Doppler (PD) signal was determined, ranging from 0 to 3 . The serum $C$ reactive protein (CRP), matrix metalloproteinase-3 (MMP-3) and DAS28 values were also examined

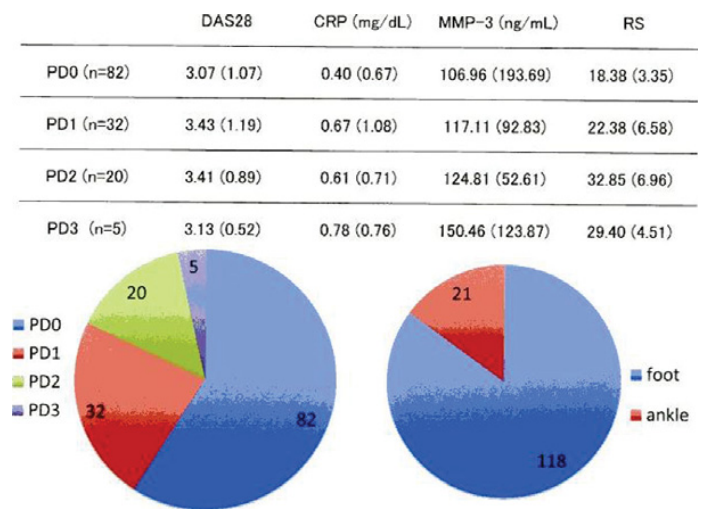

just before surgery. A histopathological examination of the gathered synovium at the surgical site was performed using the Rooney score (RS). Biological disease-modifying antirheumatic drugs were used in 45 cases, namely infliximab in 7 cases, etanercept in 16, adalimumab in 7 , tocilizumab in 6 , abatacept in 4 , certolizumab pegol in 1, and golimumab in 4 .

Results: The PD score was grade 0 in 82 cases, 1 in 32 cases, 2 in 20 cases and 3 in 5 cases. The total RS and its item scores except for "proliferating blood vessels" correlated well with the PD signal intensity. The systemic disease activity, as indicated by DAS28, CRP and MMP-3, had no significant correlation with the local PD signal intensity. However, the DAS28, CRP and MMP-3 values and each RS item score, except for "proliferating blood vessels", were significantly lower in the PD grade 0 group than in the PD grades 1, 2 and 3 groups.

Conclusions: No PD signal intensity in the ankle and foot indicates systemically well-controlled disease activity. US is an excellent tool for determining local synovitis as well as the systemic disease activity in patients with RA

Disclosure of Interest: None declared

DOI: 10.1136/annrheumdis-2017-eular.2011

\section{THU0080 DEPRESSION AND ANXIETY REDUCE THE LIKELIHOOD OF ACHIEVING REMISSION IN PATIENTS WITH RHEUMATOID ARTHRITIS: REAL LIFE DATA FROM THE NOR-DMARD STUDY}

B. Michelsen $^{1,2}$, E.K. Kristianslund ${ }^{2}$, K.M. Fageli ${ }^{2}$, E. Lie ${ }^{2}$, H.B. Hammer ${ }^{2}$, G. Haugeberg ${ }^{1,3}$, T.K. Kvien ${ }^{2} .{ }^{1}$ Dept. of Rheumatology, Hospital of Southern Norway Trust, Kristiansand; ${ }^{2}$ Dept. of Rheumatology, Diakonhjemmet Hospital, Oslo; ${ }^{3}$ Dept. of Rheumatology, Martina Hansens Hospital, Bærum, Norway

Background: Depression and anxiety are reported to predict poorer treatment outcomes in rheumatoid arthritis (RA). ${ }^{1}$ Whether this can be confirmed in larger prospective observational studies using various remission criteria remains to be explored.

Objectives: To investigate the predictive value of baseline depression/anxiety on the likelihood of achieving remission in RA as well as the associations between baseline depression/anxiety and the components of the remission criteria at follow-up.

Methods: From the prospective, multi-center NOR-DMARD study we included RA patients starting first-time tumour necrosis factor inhibitors (TNFi) and DMARD naïve RA patients starting methotrexate (MTX) between year 2006 and 2012. The following two criteria for depression/anxiety were assessed: 1) the Medical Outcomes Survey Short Form-36 (SF-36) Mental Health subscale (MH) $\leq 56$ and 2) the SF-36 Mental Component Summary score (MCS) $\leq 38 .^{2}$ The predictive value of baseline depression/anxiety on remission after 3 and 6 months treatment was explored in prespecified logistic regression models adjusted for age, sex, disease duration and smoking and the associations between baseline depression/anxiety and the different components of the remission criteria at follow-up in prespecified multiple linear regression models adjusted for age, sex, disease duration and smoking. Results: A total of 1450 RA patients were included (mean (SD) age 54.4 (13.5) years, median (25th-75th percentile) disease duration $0.4(0.0-5.0)$ years, $68.7 \%$ females and $28.6 \%$ current smokers). According to the SF- $36 \mathrm{MH} \leq 56 / \mathrm{SF}-$ $36 \mathrm{MCS} \leq 38$ criteria $18.1 / 29.9 \%$ of the patients were depressed/anxious at baseline, respectively. Lower percentages of patients with versus without baseline depression/anxiety achieved remission at 3 and 6 months treatment (unadjusted analyses, figure). Baseline depression/anxiety negatively predicted remission after 3 and 6 months (adjusted analyses, table 1 and 2 ).

The findings were confirmed in separate subgroup analyses of TNFi/MTX treated patients. Baseline depression/anxiety were associated with increased patient's and evaluator's global assessment and 28 tender joint count at 3 and 6 months, but not with level of acute phase reactants or 28 swollen joint count.

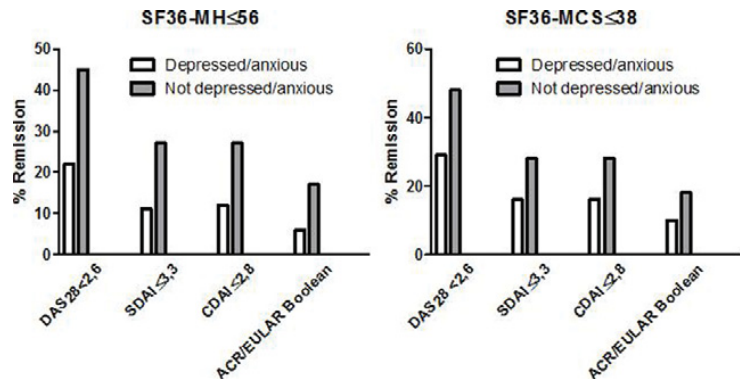

Abstract THU0080 - Table 1. Odds ratio $(95 \% \mathrm{Cl})$ for remission in patients with versus without baseline depression/anxiety according to the SF36-MH $\leq 56$ criterion

\begin{tabular}{|c|c|c|c|c|}
\hline Months & DAS28<2.6 & $\mathrm{SDAI} \leq 3.3$ & $\mathrm{CDAI} \leq 2.8$ & ACR/EULAR Boolean \\
\hline 3 & $0.48(0.32-0.73), p<0.001$ & $0.43(0.25-0.74), p=0.003$ & $0.38(0.22-0.65), p<0.001$ & $0.27(0.13-0.57), p=0.001$ \\
\hline 6 & $0.36(0.24-0.56), p<0.001$ & $0.35(0.20-0.60), p<0.001$ & $0.38(0.23-0.63), p<0.001$ & $0.31(0.16-0.61), p=0.001$ \\
\hline
\end{tabular}

Abstract THU0080 - Table 2. Odds ratio $(95 \% \mathrm{Cl})$ for remission in patients with versus without baseline depression/anxiety according to the SF36MCS $\leq 38$ criterion

\begin{tabular}{lcccc}
\hline Months & DAS28ESR $<2.6$ & SDAI $\leq 3.3$ & CDAI $\leq 2.8$ & ACR/EULAR Boolean \\
\hline 3 & $0.67(0.49-0.93), p=0.02$ & $0.51(0.34-0.78), p=0.002$ & $0.47(0.32-0.70), p<0.001$ & $0.35(0.21-0.59), p<0.001$ \\
6 & $0.45(0.32-0.63), p<0.001$ & $0.46(0.31-0.69), p<0.001$ & $0.48(0.33-0.69), p<0.001$ & $0.46(0.29-0.72), p=0.001$
\end{tabular}

